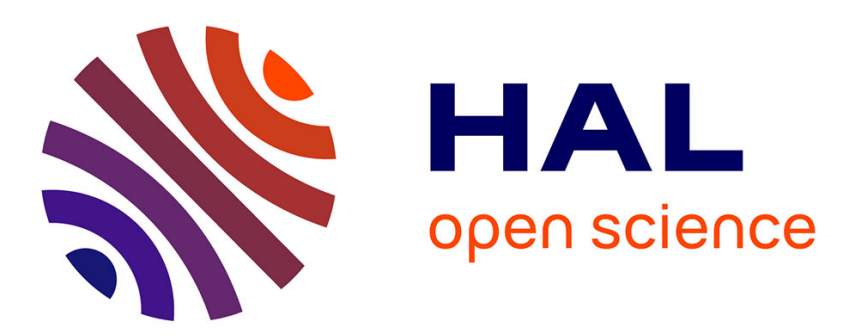

\title{
Dual formulation of a quasistatic viscoelastic contact problem with tresca's friction law
}

\author{
B. Awbl, M. Shillor, M. Sofonea
}

\section{To cite this version:}

B. Awbl, M. Shillor, M. Sofonea. Dual formulation of a quasistatic viscoelastic contact problem with tresca's friction law. Applicable Analysis, 2001, 79 (1-2), pp.1-20. 10.1080/00036810108840949 . hal-01352587

\section{HAL Id: hal-01352587 \\ https://hal.science/hal-01352587}

Submitted on 8 Aug 2016

HAL is a multi-disciplinary open access archive for the deposit and dissemination of scientific research documents, whether they are published or not. The documents may come from teaching and research institutions in France or abroad, or from public or private research centers.
L'archive ouverte pluridisciplinaire $\mathbf{H A L}$, est destinée au dépôt et à la diffusion de documents scientifiques de niveau recherche, publiés ou non, émanant des établissements d'enseignement et de recherche français ou étrangers, des laboratoires publics ou privés. 


\title{
Dual Formulation of a Quasistatic Viscoelastic Contact Problem with Tresca's Friction Law
}

\author{
B. AWBI ${ }^{a}, M \cdot$ SHILLOR ${ }^{b}$ and M. SOFONEA ${ }^{a, *}$ \\ a Laboratoire de Théorie des Systèmes, University of Perpignan, \\ 52 Avenue de Villeneuve, 66860 Perpignan, France; \\ ${ }^{\mathrm{b}}$ Department of Mathematics and Statistics, Oakland University, \\ Rochester, MI 48309, USA
}

We consider quasistatic evolution of a viscoelastic body which is in bilateral frictional contact with a rigid foundation. We derive two variational formulations for the problem: the primal formulation in terms of the displacements and the dual formulation in terms of the stress field. We prove the existence of a unique solution to each one and establish the equivalence between the two variational formulations. We also prove the continuous dependence of the solution on the friction yield limit.

Keywords: Quasistatic bilateral frictional contact; Viscoelastic constitutive law; Dual problem; Fixed point; Tresca's law of friction

AMS: 74M10; 35J85; 74D10; 74M15

\section{INTRODUCTION}

We investigate a model for the process of quasistatic frictional contact between a viscoelastic body and a rigid foundation. Processes of frictional contact are very common in industry and everyday 
life. Contact without lubrication can be found for example in breaking systems and in train wheels and tracks. Often, in practice, the main interest lies in the contact stress, since the behavior of the system and especially the surface integrity and wear depend on it. For this reason we obtain and analyze a formulation of the problem in terms of the stress, the so-called "dual formulation".

This work is a continuation of $[15,16]$ where related problems were investigated, but there only the primal formulations, in terms of the displacements, were considered.

We study the contact problem for a general viscoelastic material with constitutive law

$$
\sigma=\mathcal{A}(\varepsilon(\dot{u}))+\mathcal{G}(\varepsilon(u)),
$$

where $\sigma$ denotes the stress tensor, $u$ the displacements and $\varepsilon=\varepsilon(u)$ the linearized strain tensor. $\mathcal{A}$ and $\mathcal{G}$ are general nonlinear constitutive functions. Here and below, a dot above a variable denotes the time derivative. Such general constitutive laws were used recently in $[15,16]$.

We assume that there is no loss of contact between the body and the foundation, i.e. the contact is bilateral. The friction process is described by Tresca's law

$$
\left.\begin{array}{l}
\left|\sigma_{\tau}\right| \leq g, \\
\left|\sigma_{\tau}\right|<g \Rightarrow \dot{u}_{\tau}=0, \\
\left|\sigma_{\tau}\right|=g \Rightarrow \text { there exists } \lambda \geq 0 \text { such that } \sigma_{\tau}=-\lambda \dot{u}_{\tau},
\end{array}\right\}
$$

which takes place on the contact surface $\Gamma_{C}$, where $\dot{u}_{\tau}$ denotes the tangential velocity, $\sigma_{\tau}$ represents the tangential traction and $g$ is the friction yield limit. The strong inequality holds in the stick zone and the equality in the slip zone.

The classical formulation of the model consists of a system of evolution equations with a frictional boundary condition on the contact surface. Since, generally, such problems do not have classical solutions, we reformulate the model as a variational inequality for the displacements. This is the primal formulation. Our interest lies in obtaining and analyzing a dual formulation in terms of the stress. Indeed, as noted above, in most engineering applications the distribution of the contact stress is of greater importance than the displacements. Moreover, even when the displacements are reasonably 
accurate, in the numerical solutions of contact problems, the stress which is obtained by numerical differentiation is considerably less accurate.

In this paper we prove the existence of a unique weak solution for each one of the formulations and establish their equivalence. The existence and uniqueness result is accomplished in a number of steps, where fixed point arguments are used. From the equivalence we find that the stress in the primal problem is the solution of the dual one.

We prove the continuous dependence of the solutions on the friction yield limit $g$. This is important in applications since it indicates that small inaccuracies in $g$ lead to small variations in the solution.

A number of quasistatic contact problems with friction have been investigated recently in the literature. Constitutive laws of linear elasticity and normal compliance contact condition were used in $[4,12]$ while Sigorini's condition in [5-7]. Bilateral contact with more general constitutive laws can be found in [1-3,16], and viscoelastic contact problems with normal compliance and friction were studied in [15]. A quasistatic problem which takes thermal effects into consideration, especially the frictional heat generation, was analyzed in [14]. Except for [12], these papers deal with the primal formulation of the problem. In [12] only the dual problem was considered. Here, we deal with both formulations and their equivalence.

The rest of the paper is organized as follows. Section 2 contains notation and preliminary material. In Section 3 we describe the classical model for the process and formulate it as two equivalent variational inequalities, problems $P_{1}$ and $P_{2}$. Problem $P_{1}$, the primal one, is an evolution variational inequality in which the unknown is the displacement field $u$. Problem $P_{2}$, the dual problem, is an inequality in terms of the stress field $\sigma$. We establish the existence and uniqueness of the solution for each of the problems in Section 4 by using arguments from the theory of elliptic variational inequalities and a fixed point theorem. In Section 5 we prove the equivalence of the formulations in the sense that if $u$ is the unique solution of $P_{1}$ and stress field $\sigma$ is the solution of $P_{2}$, then $u$ and $\sigma$ are related by the viscoelastic constitutive law (1.1). Finally, in Section 6 we show the continuous dependence of the solutions of problems $P_{1}$ and $P_{2}$ on the friction yield limit $g$. 


\section{NOTATION AND PRELIMINARIES}

In this short section we present the notations and some preliminary material we shall use, and for further details we refer the reader to $[8,10,11]$ or [13]. We denote by $S_{M}$ the space of second order symmetric tensors on $\mathbb{R}^{M}(M=2,3)$, while "." and $|\cdot|$ represent the inner product and the Euclidean norm on $S_{M}$ and $\mathbb{R}^{M}$, respectively. Let $\Omega \subset \mathbb{R}^{M}$ be a bounded domain with a Lipschitz boundary $\Gamma$ and let $\nu$ denote the unit outer normal on $\Gamma$. We let

$$
\begin{aligned}
H & =\left\{u=\left(u_{i}\right) \mid u_{i} \in L^{2}(\Omega)\right\}, \quad \mathcal{H}=\left\{\sigma=\left(\sigma_{i j}\right) \mid \sigma_{i j}=\sigma_{j i} \in L^{2}(\Omega)\right\}, \\
H_{1} & =\left\{u=\left(u_{i}\right) \mid u_{i} \in H^{1}(\Omega)\right\}, \quad \mathcal{H}_{1}=\left\{\sigma \in \mathcal{H} \mid \sigma_{i j, j} \in H\right\},
\end{aligned}
$$

where and below $i, j=1, \ldots, M$, summation over repeated indices is implied and the index that follows a comma indicates a partial derivative. $H, \mathcal{H}, H_{1}$ and $\mathcal{H}_{1}$ are real Hilbert spaces endowed with the inner products given by

$$
\langle u, v\rangle_{H}=\int_{\Omega} u_{i} v_{i} d x, \quad\langle\sigma, \tau\rangle_{\mathcal{H}}=\int_{\Omega} \sigma_{i j} \tau_{i j} d x,
$$

and

$$
\begin{aligned}
\langle u, v\rangle_{H_{1}} & =\langle u, v\rangle_{H}+\left\langle\varepsilon(u),(\varepsilon(v)\rangle_{\mathcal{H}},\right. \\
\langle\sigma, \tau\rangle_{\mathcal{H}_{1}} & =\langle\sigma, \tau\rangle_{\mathcal{H}}+\langle\operatorname{div} \sigma, \operatorname{div} \tau\rangle_{H},
\end{aligned}
$$

respectively. Here $\varepsilon: H_{1} \rightarrow \mathcal{H}$ and div $: \mathcal{H}_{1} \rightarrow H$ are the strain and the divergence operators, respectively, defined by

$$
\varepsilon(v)=\left(\varepsilon_{i j}(v)\right), \quad \varepsilon_{i j}(v)=\frac{1}{2}\left(v_{i, j}+v_{j, i}\right), \quad \operatorname{div} \sigma=\left(\sigma_{i j, j}\right) .
$$

The associated norms on the space $H, \mathcal{H}, H_{1}$ and $\mathcal{H}_{1}$ are denoted by $|\cdot|_{H},|\cdot|_{\mathcal{H}},|\cdot|_{H_{1}}$ and $|\cdot|_{\mathcal{H}_{1}}$, respectively. Let $H_{\Gamma}=H^{1 / 2}(\Gamma)^{M}$ and let $\gamma: H_{1} \rightarrow H_{\Gamma}$ be the trace map. For every element $v \in H_{1}$ we use, when no confusion is likely, the notation $v$ for the trace $\gamma v$ of $v$ on $\Gamma$ and we denote by $v_{\nu}=v \cdot v$ and $v_{\tau}=\nu-v_{\nu} \cdot \nu$ the normal and the tangential components of $v$ on $\Gamma$, respectively.

Let $H_{\Gamma}^{\prime}$ be the dual of $H_{\Gamma}$ and let $\langle\cdot, \cdot\rangle$ denote the duality pairing between $H_{\Gamma}^{\prime}$ and $H_{\Gamma}$. For every $\sigma \in \mathcal{H}_{1}$, let $\sigma v$ be the element of $H_{\Gamma}^{\prime}$ 
given by

$$
\langle\sigma v, \gamma v\rangle_{H_{\Gamma}^{\prime} \times H_{\Gamma}}=\left\langle\sigma,\left.\varepsilon(v)\right|_{\mathcal{H}}+\langle\operatorname{div} \sigma, v\rangle_{H} \quad \forall v \in H_{1}\right.
$$

We also denote by $\sigma_{v}$ and $\sigma_{\tau}$ the normal and tangential traces of $\sigma$ (see, e.g., [13]). We recall that if $\sigma$ is a regular function, then

$$
\langle\sigma v, \gamma v\rangle=\int_{\Gamma} \sigma v \cdot v d a
$$

for all $v \in H_{1}$, where $d a$ is the surface measure element, and

$$
\sigma_{\nu}=(\sigma \nu) \cdot \nu, \quad \sigma_{\tau}=\sigma \nu-\sigma_{\nu} \nu .
$$

Finally, let $\left(X,|\cdot|_{X}\right)$ be a real normed space, then $C(0, T ; X)$ and $C^{1}(0, T ; X)$ denote the spaces of continuous and continuously differentiable functions from $[0, T]$ to $X$, with norms

$$
|f|_{C(0, T ; X)}=\max _{t \in[0, T]}|f(t)|_{X}, \quad|f|_{C^{1}(0, T ; X)}=\max _{t \in[0, T]}\left\{|f(t)|_{X}+|\dot{f}(t)|_{X}\right\}
$$

respectively.

\section{THE PROBLEM AND VARIATIONAL FORMULATION}

The physical setting we consider is as follows. A viscoelastic body occupies the domain $\Omega$ and has surface $\Gamma$ that is partitioned into three disjoint measurable parts $\Gamma_{D}, \Gamma_{N}$ and $\Gamma_{C}$ such that meas $\Gamma_{D}>0$. The body is clamped on $\Gamma_{D} \times(0, T)$ and the displacements vanish there. Surface tractions $\mathrm{f}_{N}$ act on $\Gamma_{D} \times(0, T)$. The solid is in bilateral frictional contact with a rigid foundation on $\Gamma_{C} \times(0, T)$, which means that the body and foundation have a compliant shape on $\Gamma_{C}$ and there is no loss of contact. A volume force of density $f_{0}$ is applied in $\Omega \times(0, T)$. We are interested in the evolution of the frictional process on the time interval $[0, T]$, for $T>0$. Assuming the constitutive law (1.1), Tresca's law of friction (1.2) and slow evolution of $\mathrm{f}_{0}$ and $\mathrm{f}_{N}$, the classical formulation of the mechanical problem is the following. 
Problem $P$ : Find a displacement field $u: \Omega \times[0, T] \rightarrow \mathbb{R}^{M}$ and a stress field $\sigma: \Omega \times[0, T] \rightarrow S_{M}$ such that

$$
\begin{gathered}
\sigma=\mathcal{A}(\varepsilon(\dot{u}))+\mathcal{G}(\varepsilon(u)) \quad \text { in } \Omega \times(0, T), \\
\operatorname{div} \sigma+\mathrm{f}_{0}=0 \quad \text { in } \Omega \times(0, T), \\
u=0 \quad \text { on } \Gamma_{D} \times(0, T), \\
\sigma v=\mathrm{f}_{N} \quad \text { on } \Gamma_{N} \times(0, T), \\
\left.\begin{array}{c}
u_{v}=0,\left|\sigma_{\tau}\right| \leq g \quad \text { on } \Gamma_{C} \times(0, T), \\
\left|\sigma_{\tau}\right|<g \Rightarrow \dot{u}_{\tau}=0, \\
\left|\sigma_{\tau}\right|=g \Rightarrow \text { there exists } \lambda \geq 0 \text { such that } \sigma_{\tau}=-\lambda \dot{u}_{\tau},
\end{array}\right\} \\
u(0)=u_{0} \text { in } \Omega .
\end{gathered}
$$

Here (3.2) is the quasistatic equation of motion, since the inertial terms have been omitted, and $u_{0}$ is the initial displacement field.

To describe the two varational formulations of problem (3.1)-(3.6), we need additional notation. Let $V$ denote the closed subspace of $H_{1}$ given by

$$
V=\left\{v \in H_{1} \mid v=0 \text { on } \Gamma_{D}, v_{\nu}=0 \text { on } \Gamma_{C}\right\} .
$$

Since meas $\Gamma_{D}>0$, Korn's inequality holds (see, e.g., [9] p. 79), thus

$$
|\varepsilon(v)|_{\mathcal{H}} \geq C|v|_{H_{1}} \quad \forall v \in V .
$$

Here and below, $C$ denotes a positive generic constant which may depend on $\Omega, \Gamma, \mathcal{A}, \mathcal{G}$ and $T$ but does not depend on time or on the input data $\mathrm{f}_{0}, \mathrm{f}_{N}, u_{0}$ and $g$, and whose value may vary form place to place.

On $V$ we use the inner product

$$
\langle u, v\rangle_{V}=\langle\varepsilon(u), \varepsilon(v)\rangle_{\mathcal{H}},
$$

and it follows from (3.7) that $|\cdot|_{H_{1}}$ and $|\cdot|_{V}$ are equivalent norms on $V$. Therefore, $\left(V,|\cdot|_{V}\right)$ is a real Hilbert space.

In the study of the mechanical problem (3.1)-(3.6) we assume that

$$
\mathcal{A}: \Omega \times S_{M} \rightarrow S_{M},
$$


and

(a) there exists $L>0$ such that

$$
\left|\mathcal{A}\left(x ; \varepsilon_{1}\right)-\mathcal{A}\left(x ; \varepsilon_{2}\right)\right| \leq L\left|\varepsilon_{1}-\varepsilon_{2}\right| \quad \forall \varepsilon_{1}, \varepsilon_{2} \in S_{M}, \text { a.e. in } \Omega \text {; }
$$

(b) there exists $m>0$ such that

$$
\mathcal{A}\left(x, \varepsilon_{1}\right)-\mathcal{A}\left(x ; \varepsilon_{2}\right) \cdot\left(\varepsilon_{1}-\varepsilon_{2}\right) \geq m\left|\varepsilon_{1}-\varepsilon_{2}\right|^{2} \forall \varepsilon_{1}, \varepsilon_{2} \in S_{M} \text {, a.e. in } \Omega \text {; }
$$

(c) $x \rightarrow \mathcal{A}(x ; \varepsilon)$ is Lebesgue measurable on $\Omega$, for all $\varepsilon \in S_{M}$;

(d) $x \rightarrow \mathcal{A}(x ; 0) \in \mathcal{H}$.

Also

$$
\mathcal{G}: \Omega \times S_{M} \rightarrow S_{M}
$$

and

(a) there exists $\tilde{L}>0$ such that

$\left|\mathcal{G}\left(x ; \varepsilon_{1}\right)-\mathcal{G}\left(x ; \varepsilon_{2}\right)\right| \leq \tilde{L}\left|\varepsilon_{1}-\varepsilon_{2}\right| \quad \forall \varepsilon_{1}, \varepsilon_{2} \in S_{M}, \quad$ a.e. in $\Omega$;

(b) $x \rightarrow \mathcal{G}(x ; \varepsilon)$ is Lebesgue measurable on $\Omega$, for all $\varepsilon \in S_{M}$;

(c) $x \rightarrow \mathcal{G}(x ; 0) \in \mathcal{H}$.

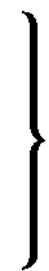

We obtain from (3.9) that for $\tau \in \mathcal{H}$ the function $x \rightarrow \mathcal{A}(x ; \tau(x))$ belongs to $\mathcal{H}$ and hence we may consider $\mathcal{A}$ as an operator on $\mathcal{H}$ with range in $\mathcal{H}$. Moreover, $\mathcal{A}: \mathcal{H} \rightarrow \mathcal{H}$ is a strongly monotone Lipschitz continuous operator and, therefore, $\mathcal{A}$ is invertible and its inverse $\mathcal{A}^{-1}: \mathcal{H} \rightarrow \mathcal{H}$ is also a strongly monotone Lipschitz continuous operator. Similar arguments allow us to consider $\mathcal{G}: \mathcal{H} \rightarrow \mathcal{H}$ as a Lipschitz continuous operator, too. We assume in addition that

$$
\begin{gathered}
\mathrm{f}_{0} \in C(0, T ; H), \\
\mathrm{f}_{N} \in C\left(0, T ; L^{2}\left(\Gamma_{N}\right)^{M}\right),
\end{gathered}
$$$$
g \in L^{\infty}\left(\Gamma_{C}\right) \text { and } g(x) \geq 0 \text { a.e. on } \Gamma_{C} \text {, }
$$

$$
u_{0} \in V \text {. }
$$

Let $f(t)$ be the element of $V$ given by

$$
\langle f(t), v\rangle_{V}=\left\langle\mathrm{f}_{0}(t), v\right\rangle_{H}+\left\langle\mathrm{f}_{N}(t), \gamma v\right\rangle_{L^{2}\left(\Gamma_{N}\right)^{M}},
$$


for all $v \in V$ and for all $t \in[0, T]$. We note that (3.11) and (3.12) imply

$$
f \in C(0, T ; V)
$$

Let $j$ be a continuous seminorm on $V$ given by

$$
j(v)=\int_{\Gamma_{C}} g\left|v_{\tau}\right| d a \quad \forall v \in V .
$$

By (3.13) the integral is well defined and, moreover,

$$
j(v) \leq C|g|_{L^{\infty}\left(\Gamma_{C}\right)}|v|_{V} \quad \forall v \in V .
$$

Finally, for each $t \in[0, T]$, the set $\Sigma(t)$ is given by

$$
\Sigma(t)=\left\{\tau \in \mathcal{H} \mid\langle\tau, \varepsilon(v)\rangle_{\mathcal{H}}+j(v) \geq\langle f(t), v\rangle_{V} \quad \forall v \in V\right\} .
$$

Now, we obtain from (3.15)

$$
\tau \in \Sigma(t) \Rightarrow \operatorname{div} \tau+\mathrm{f}_{0}(t)=0 \quad \text { in } \Omega, \quad t \in[0, T] .
$$

To proceed we need the following results.

LEMMA 3.1 If the pair of functions $\{u, \sigma\}$ is a regular solution of (3.1)(3.6), then

$$
\left.\langle\sigma(t), \varepsilon(w)-\varepsilon(\dot{u}(t))\rangle_{\mathcal{H}}+j(w)-j(\dot{u}(t)) \geq\langle f(t), w-\dot{u}(t))\right\rangle_{V} \quad \forall w \in V,
$$

and

$$
\sigma(t) \in \Sigma(t), \quad\left\langle\tau-\sigma(t),\left.\varepsilon(\dot{u}(t))\right|_{\mathcal{H}} \geq 0 \quad \forall \tau \in \Sigma(t),\right.
$$

for each $t \in[0, T]$.

Proof Let $w \in V$. Using (2.1), (2.2) and (3.2) for each $t \in[0, T]$ we have

$$
\langle\sigma(t), \varepsilon(w)-\varepsilon(\dot{u}(t))\rangle_{\mathcal{H}}=\left\langle\mathrm{f}_{0}(t), w-\dot{u}(t)\right\rangle_{H}+\int_{\Gamma} \sigma(t) v(w-\dot{u}(t)) d a,
$$

and using (3.3), (3.4) and (3.15) we obtain

$$
\langle\sigma(t), \varepsilon(w)-\varepsilon(\dot{u}(t))\rangle_{\mathcal{H}}=\langle f(t), w-\dot{u}(t)\rangle_{V}+\int_{\Gamma_{C}} \sigma(t) v(w-\dot{u}(t)) d a .
$$


Moreover, from (2.2), (3.5) and (3.17) we get

$$
\int_{\Gamma_{c}} \sigma(t) v(w-\dot{u}(t)) d a \geq j(\dot{u}(t))-j(w) .
$$

The inequality (3.21) follows now from (3.24) and (3.25). We now chose $w=2 \dot{u}(t)$ and $w=0$ in (3.21), both in $V$, and obtain

$$
\langle\sigma(t), \varepsilon(\dot{u}(t))\rangle_{\mathcal{H}}+j(\dot{u}(t))=\langle f(t), \dot{u}(t)\rangle_{V} .
$$

Using now (3.26) and (3.21) we find that $\sigma(t) \in \Sigma(t)$, for each $t \in[0, T]$. The inequality in (3.22) follows from (3.19) and (3.26).

LemMA 3.2 Let $\sigma \in C(0, T ; \mathcal{H})$ and $z_{0} \in \mathcal{H}$, then there exists a unique function $z \in C(0, T ; \mathcal{H})$ such that

$$
\sigma(t)=\mathcal{A}(z(t))+\mathcal{G}\left(\int_{0}^{t} z(s) d s+z_{0}\right) \quad \forall t \in[0, T] .
$$

Proof Let $\sigma \in C(0, T ; \mathcal{H})$ and $z_{0} \in \mathcal{H}$. We may consider the operator

$$
\mathcal{T}: C(0, T ; \mathcal{H}) \rightarrow C(0, T ; \mathcal{H}),
$$

defined by

$$
\mathcal{T} z(t)=\mathcal{A}^{-1}(\sigma(t))-\mathcal{A}^{-1} \mathcal{G}\left(\int_{0}^{t} z(s) d s+z_{0}\right),
$$

for $z \in C(0, T ; \mathcal{H})$ and $t \in[0, T]$. It is straightforward to show that $z$ satisfies (3.27) if and only if $z$ is a fixed point of operator $\mathcal{T}$. From (3.28) we obtain

$$
\left|\mathcal{T}_{z_{1}}(t)-\mathcal{T} z_{2}(t)\right|_{\mathcal{H}} \leq C \int_{0}^{t}\left|z_{1}(s)-z_{2}(s)\right|_{\mathcal{H}} d s,
$$

for all $t \in[0, T]$ and $z_{1}, z_{2} \in C(0, T ; \mathcal{H})$. Reiterating this inequality $n$ times yields

$$
\left|\mathcal{T}^{n} z_{1}(t)-\mathcal{T}^{n} z_{2}(t)\right|_{C(0, T ; \mathcal{H})} \leq \frac{C^{n}}{n !}\left|z_{1}-z_{2}\right|_{C(0, T: \mathcal{H})}
$$

which implies that for $n$ sufficiently large $\mathcal{T}^{n}$, a power of $\mathcal{T}$, is a contraction on $C(0, T ; \mathcal{H})$. Then, it follows from the Banach theorem that $\mathcal{T}$ has a unique fixed point, which concludes the proof. 
The lemmas allow us to define the operator $B: C(0, T ; \mathcal{H}) \times$ $\mathcal{H} \rightarrow C(0, T ; \mathcal{H})$ by $B\left(\sigma, z_{0}\right)=z$. Using $(3.27)$ we have

$B\left(\sigma, z_{0}\right)=z \Longleftrightarrow z(t)=\mathcal{A}^{-1}(\sigma(t))-\mathcal{A}^{-1} \mathcal{G}\left(\int_{0}^{t} z(s) d s+z_{0}\right) \quad \forall t \in[0, T]$,

which, for each $t \in[0, T]$, implies

$$
B\left(\sigma, z_{0}\right)(t)=\mathcal{A}^{-1}(\sigma(t))-\mathcal{A}^{-1} \mathcal{G}\left(\int_{0}^{t} B\left(\sigma, z_{0}\right) d s+z_{0}\right) .
$$

Using (3.9), (3.10), (3.32) and standard arguments, we deduce that $B$ is a Lipschitz continuous operator and there exists a positive constant $C$ such that

$$
\left|B\left(\sigma_{1}, z_{01}\right)-B\left(\sigma_{2}, z_{02}\right)\right|_{C(0, T ; \mathcal{H})} \leq C\left(\left|\sigma_{1}-\sigma_{2}\right|_{C(0, T ; \mathcal{H})}+\left|z_{01}-z_{02}\right|_{\mathcal{H}}\right),
$$

for all $\sigma_{1}, \sigma_{2} \in C(0, T ; \mathcal{H})$ and $z_{01}, z_{02} \in \mathcal{H}$.

From (3.1), (3.6) and (3.21) we obtain the primal formulation of problem $P$.

Problem $P_{1}:$ Find the displacement field $u:[0, T] \rightarrow V$ such that

$$
\begin{aligned}
& \langle\mathcal{A}(\varepsilon(\dot{u}(t))), \varepsilon(w)-\varepsilon(\dot{u}(t))\rangle_{\mathcal{H}}+\langle\mathcal{G}(\varepsilon(u(t))), \varepsilon(w)-\varepsilon(\dot{u}(t))\rangle_{\mathcal{H}} \\
& \quad+j(w)-j(\dot{u}(t)) \geq\langle f(t), w-\dot{u}(t)\rangle_{V} \quad \forall w \in V,
\end{aligned}
$$

for each $t \in[0, T]$, and

$$
u(0)=u_{0}
$$

Using now (3.1), (3.6) and (3.31) we find that $\varepsilon(\dot{u})=B\left(\sigma, \varepsilon\left(u_{0}\right)\right)$, and by (3.22) we obtain the dual variational formulation of problem $P$.

Problem $P_{2}:$ Find the stress field $\sigma:[0, T] \rightarrow \mathcal{H}$ such that

$$
\sigma(t) \in \Sigma(t), \quad\left\langle B\left(\sigma, \varepsilon\left(u_{0}\right)\right)(t), \tau-\left.\sigma(t)\right|_{\mathcal{H}} \geq 0 \quad \forall \tau \in \Sigma(t), t \in[0, T] .\right.
$$

We conclude that if $\{u, \sigma\}$ is a regular solution of the mechanical problem $P$ then $u$ is a solution of problem $P_{1}$ and $\sigma$ is a solution of problem $P_{2}$. For this reason we refer to problems $P_{1}$ and $P_{2}$ as 
variational formulations of problem $P$. We will show in Section 5 that these formulations are equivalent.

\section{EXISTENCE AND UNIQUENESS}

In this section we state and prove the existence and uniqueness of solutions of the variational problems $P_{1}$ and $P_{2}$. Our first result is:

THEOREM 4.1 Let (3.9)-(3.14) hold. Then there exists a unique solution $u \in C^{1}(0, T ; V)$ of problem $P_{1}$.

The proof of the theorem is based on fixed point arguments and will be carried out in several steps. Similar ideas were used in [1,15] but there the setting was different and so was the choice of the operators. To simplify the notation we shall not indicate explicitly the dependence on $t$.

Let $\eta \in C(0, T ; \mathcal{H})$, and consider the following variational problem. Problem $P_{1 \eta}:$ Find $v_{\eta}:[0, T] \rightarrow V$ such that

$$
\begin{aligned}
& \left\langle\mathcal{A}\left(\varepsilon\left(v_{\eta}(t)\right)\right), \varepsilon(w)-\varepsilon\left(v_{\eta}(t)\right)\right\rangle_{\mathcal{H}}+\left\langle\eta(t), \varepsilon(w)-\varepsilon\left(v_{\eta}(t)\right)\right\rangle_{\mathcal{H}} \\
& \quad+j(w)-j\left(v_{\eta}(t)\right) \geq\left\langle f(t), w-v_{\eta}(t)\right\rangle_{V} \quad \forall w \in V, t \in[0, T] .
\end{aligned}
$$

Lemma 4.2 Problem $P_{1 \eta}$ has a unique solution $v_{\eta} \in C(0, T ; V)$.

Proof Let $t \in[0, T]$. It follows from classical results for elliptic variational inequalities that there exists a unique element $v_{\eta}(t) \in V$ which is a solution of (4.1). Now, let $t_{1}, t_{2} \in[0, T]$, and for the sake of simplicity we denote $v_{\eta}\left(t_{i}\right)=v_{i}, \eta\left(t_{i}\right)=\eta_{i}, f\left(t_{i}\right)=f_{i}$ for $i=1,2$. Using (4.1) we find

$$
\begin{aligned}
& \left\langle\mathcal{A}\left(\varepsilon\left(v_{1}\right)\right)-\mathcal{A}\left(\varepsilon\left(v_{2}\right)\right), \varepsilon\left(v_{1}\right)-\varepsilon\left(v_{2}\right)\right\rangle_{\mathcal{H}} \leq\left\langle f_{1}-f_{2}, v_{1}-v_{2}\right\rangle_{V} \\
& \quad-\left\langle\eta_{1}-\eta_{2}, \varepsilon\left(v_{1}\right)-\varepsilon\left(v_{2}\right)\right\rangle_{\mathcal{H}}
\end{aligned}
$$

and by using (3.9) and Korn's inequality (3.7) we get

$$
\left|v_{1}-v_{2}\right|_{V} \leq C\left(\left|f_{1}-f_{2}\right|_{V}+\left|\eta_{1}-\eta_{2}\right|_{\mathcal{H}}\right) .
$$


The regularity property $v_{\eta} \in C(0, T ; V)$ follows from (4.2), since $f \in C(0, T ; V)$ and $\eta \in C(0, T ; \mathcal{H})$.

Let now $u_{n}:[0, T] \rightarrow V$ be the function

$$
u_{\eta}(t)=u_{0}+\int_{0}^{t} v_{\eta}(s) d s \quad t \in[0, T]
$$

We define the operator $\Lambda: C(0, T ; \mathcal{H}) \rightarrow C(0, T ; \mathcal{H})$ by

$$
\Lambda \eta(t)=\mathcal{G}\left(\varepsilon\left(u_{\eta}(t)\right)\right)
$$

for $\eta \in C(0, T ; \mathcal{H})$ and $t \in[0, T]$.

Lemma 4.3 The operator $\Lambda$ has a unique fixed point $\eta^{*} \in C(0, T ; \mathcal{H})$. Proof Let $\eta_{1}, \eta_{2} \in C(0, T ; \mathcal{H})$ and $t \in[0, T]$. Using (3.10), (4.3) and (4.4) it follows that

$$
\left|\Lambda \eta_{1}(t)-\Lambda \eta_{2}(t)\right|_{\mathcal{H}} \leq C \int_{0}^{t}\left|v_{\eta_{1}}(s)-v_{\eta_{2}}(s)\right|_{V} d s .
$$

Moreover, we obtain from (4.2) that $\left|v_{\eta_{1}}(s)-v_{\eta_{2}}(s)\right|_{\mathcal{H}} \leq C \mid \eta_{1}(s)-$ $\left.\eta_{2}(s)\right|_{\mathcal{H}}$ for $s \in[0, T]$. Therefore,

$$
\left|\Lambda \eta_{1}(t)-\Lambda \eta_{2}(t)\right|_{\mathcal{H}} \leq C \int_{0}^{t}\left|\eta_{1}(s)-\eta_{2}(s)\right|_{\mathcal{H}} d s .
$$

Lemma 4.3 is now a consequence of (4.5) when we use the same iterative argument as in the proof of Lemma 3.2 (see (3.30)).

We have now all that is needed to prove the theorem.

Proof of Theorem 4.1 Let $\eta^{*} \in C(0, T ; \mathcal{H})$ be the fixed point of $\Lambda$ and let $v_{\eta^{*}}$ be the solution of problem $P_{1 \eta}$ for $\eta=\eta^{*}$. We show that the function $u_{\eta^{*}}$, given in (4.3), is a solution of $P_{1}$. Indeed, we get from (4.3) that $u_{\eta^{*}}$ belongs to $C^{1}(0, T ; \mathcal{H})$ and (3.35). Moreover, since $\dot{u}_{\eta^{*}}=v_{\eta^{*}}$ and $\mathcal{G}\left(\varepsilon\left(u_{\eta^{*}}\right)\right)=\Lambda \eta^{*}$, by using (4.1) we deduce that $u_{\eta^{*}}$ satisfies (3.34), which concludes the existence part in Theorem 4.1. The uniqueness follows from (3.34), (3.35) and standard estimates.

Our second result relates to the dual problem $P_{2}$. 
THEOREM 4.4 Let (3.9)-(3.14) hold. Then there exists a unique solution $\sigma \in C\left(0, T ; \mathcal{H}_{1}\right)$ of problem $P_{2}$.

The proof of Theorem 4.4 is similar to that of Theorem 4.1. Let $\eta \in C(0, T ; \mathcal{H})$, and consider the following auxiliary problem.

Problem $P_{2 \eta}$ Find $\sigma_{\eta}:[0, T] \rightarrow \mathcal{H}_{1}$ such that, for each $t \in[0, T]$,

$$
\sigma_{\eta}(t) \in \Sigma(t), \quad\left\langle\mathcal{A}^{-1}\left(\sigma_{\eta}(t)\right), \tau-\left.\sigma_{\eta}(t)\right|_{\mathcal{H}} \geq\left\langle\eta(t), \tau-\left.\sigma_{\eta}(t)\right|_{\mathcal{H}} \quad \forall \tau \in \Sigma(t)\right.\right.
$$

LemMa 4.5 Problem $P_{2 \eta}$ has a unique solution $\sigma_{\eta} \in C\left(0, T ; \mathcal{H}_{1}\right)$.

Proof The set $\Sigma(t)$ depends on time and to circumvent this fact we replace (4.6) by a variational inequality associated with a fixed convex set. To that end let

$$
\Sigma_{0}=\left\{\tau \in \mathcal{H} \mid\langle\tau, \varepsilon(v)\rangle_{\mathcal{H}}+j(v) \geq 0 \quad \forall v \in V\right\},
$$

and let $\tilde{\sigma}=\varepsilon(f)$, and set $\bar{\sigma}_{\eta}=\sigma_{\eta}-\tilde{\sigma}$. From (3.8), (3.19) and (4.7) we have

$$
\tau \in \Sigma(t) \Longleftrightarrow \tau-\tilde{\sigma} \in \Sigma_{0},
$$

for each $t \in[0, T]$ and using (3.16) we deduce

$$
\tilde{\sigma} \in C(0, T ; \mathcal{H})
$$

Therefore, by (4.7)-(4.9) it is straightforward to show that $\sigma_{\eta}$ is a solution for (4.6), such that $\sigma_{\eta} \in C(0, T ; \mathcal{H})$, if and only if $\bar{\sigma}_{\eta} \in$ $C(0, T ; \mathcal{H})$ and

$$
\begin{aligned}
\bar{\sigma}_{\eta}(t) \in \Sigma_{0}(t), \quad\left\langle A^{-1}\left(\bar{\sigma}_{\eta}(t)\right), \tau-\bar{\sigma}_{\eta}(t)\right\rangle_{\mathcal{H}} \\
\\
\geq\left\langle\eta(t)-\mathcal{A}^{-1}(\tilde{\sigma}(t)), \tau-\bar{\sigma}_{\eta}(t)\right\rangle_{\mathcal{H}} \quad \forall \tau \in \Sigma_{0},
\end{aligned}
$$

for each $t \in[0, T]$. Using standard arguments from the theory of elliptic variational inequalities we find that there exists an unique element $\bar{\sigma}_{\eta}(t) \in \mathcal{H}$ which is a solution of (4.10). 
Let now $t_{1}, t_{2} \in[0, T]$ and set $\bar{\sigma}_{\eta}\left(t_{i}\right)=\bar{\sigma}\left(t_{i}\right), \tilde{\sigma}\left(t_{i}\right)=\tilde{\sigma}_{i}, \eta\left(t_{i}\right)=\eta_{i}$, $i=1,2$. Using $(4.10)$ we get

$$
\left|\bar{\sigma}_{1}-\bar{\sigma}_{2}\right|_{\mathcal{H}} \leq C\left(\left|\eta_{1}-\eta_{2}\right|_{\mathcal{H}}+\left|\tilde{\sigma}_{1}-\tilde{\sigma}_{2}\right|_{\mathcal{H}}\right) .
$$

Therefore, by (4.9) and (4.11), we deduce that $\bar{\sigma}_{\eta} \in C(0, T ; \mathcal{H})$. We conclude that (4.6) has a unique solution $\sigma_{\eta} \in C(0, T ; \mathcal{H})$. Moreover, using (3.20) and (3.11) we deduce $\operatorname{div} \sigma_{\eta} \in C(0, T ; \mathcal{H})$ which implies $\sigma_{\eta} \in C^{1}\left(0, T ; \mathcal{H}_{1}\right)$.

We now define the operator $\Theta: C(0, T ; \mathcal{H}) \rightarrow C(0, T ; \mathcal{H})$ by

$$
\Theta \eta(t)=A^{-1} \mathcal{G}\left(\int_{0}^{t} B\left(\sigma_{\eta}, \varepsilon\left(u_{0}\right)\right)(s) d s+\varepsilon\left(u_{0}\right)\right)
$$

for $\eta \in C(0, T ; \mathcal{H})$ and $t \in[0, T]$ where $\sigma_{\eta}$ denotes the solution of (4.6) and $B$ is given in (3.31).

LeMma 4.6 The operator $\Theta$ has a unique fixed point $\eta^{*} \in C(0, T ; \mathcal{H})$.

Proof Let $\eta_{1}, \eta_{2} \in C(0, T ; \mathcal{H})$ and let $\sigma_{i}=\sigma_{\eta_{i}}, i=1,2$. Using (3.9), (3.10) and (4.12) we have

$$
\left|\Theta \eta_{1}(t)-\Theta \eta_{2}(t)\right|_{\mathcal{H}} \leq C \int_{0}^{t} \mid\left(B\left(\sigma_{1}, \varepsilon\left(u_{0}\right)\right)(s)-\left(B\left(\sigma_{2}, \varepsilon\left(u_{0}\right)\right)(s)\right) \mid d s\right.
$$

Reiterating (4.13) $n$ times we obtain

$$
\left|\Theta^{n} \eta_{1}-\Theta^{n} \eta_{2}\right|_{C(0, T ; \mathcal{H})} \leq \frac{C^{n}}{n !} \mid\left(B\left(\sigma_{1}, \varepsilon\left(u_{0}\right)\right)-\left.B\left(\sigma_{2}, \varepsilon\left(u_{0}\right)\right)\right|_{C(0, T ; \mathcal{H})} .\right.
$$

Moreover, by (3.33) and (4.10) it follows that

$$
\begin{aligned}
\left|B\left(\sigma_{1}, \varepsilon\left(u_{0}\right)\right)-B\left(\sigma_{2}, \varepsilon\left(u_{0}\right)\right)\right|_{C(0, T ; \mathcal{H})} & \leq C\left|\sigma_{1}-\sigma_{2}\right|_{C(0, T ; \mathcal{H})} \\
& \leq C\left|\eta_{1}-\eta_{2}\right|_{C(0, T ; \mathcal{H})} .
\end{aligned}
$$

Lemma 4.6 is now a consequence of (4.14) and (4.15).

Proof of Theorem 4.4 Let $\eta^{*} \in C(0, T ; \mathcal{H})$ be the fixed point of $\Theta$ and let $\sigma_{\eta^{*}}$ be the solution of $P_{2 \eta}$ for $\eta=\eta^{*}$. We show that $\sigma_{\eta^{*}}$ is a solution of Problem $P_{2}$. Indeed, by (4.12) we have

$$
\eta^{*}(t)=\Theta \eta^{*}(t)=\mathcal{A}^{-1} \mathcal{G}\left(\int_{0}^{t} B\left(\sigma_{\eta^{*}}, \varepsilon\left(u_{0}\right)\right)(s) d s+\varepsilon\left(u_{0}\right)\right) \quad t \in[0, T]
$$


and therefore, by (3.32) we find

$$
\begin{aligned}
\mathcal{A}^{-1}\left(\sigma_{\eta^{*}}(t)\right)-\eta^{*}(t) & =\mathcal{A}^{-1}\left(\sigma_{\eta^{*}}(t)\right)-\mathcal{A}^{-1} \mathcal{G}\left(\int_{0}^{t} B\left(\sigma_{\eta^{*}}, \varepsilon\left(u_{0}\right)\right)(s) d s+\varepsilon\left(u_{0}\right)\right) \\
& =B\left(\sigma_{\eta^{*}}, \varepsilon\left(u_{0}\right)\right)(t) .
\end{aligned}
$$

The existence part in Theorem 4.4 follows now from (4.6) and (4.16), while the uniqueness part results from the uniqueness of the fixed point of the operator $\Theta$.

\section{EQUIVALENCE}

In this section we study the link between the solutions $u$ and $\sigma$ of the problems $P_{1}$ and $P_{2}$, respectively. The main result of this section is that problems $P_{1}$ and $P_{2}$ are equivalent formulations of the mechanical problem $P$.

THEOREM 5.1 Let condition (3.9)-(3.14) hold.

i) If $u$ is the solution of Problem $P_{1}$ and $\sigma$ is the function given by

$$
\sigma=\mathcal{A}(\varepsilon(\dot{u}))+\mathcal{G}(\varepsilon(u))
$$

then $\sigma$ is a solution of $P_{2}$ and $\sigma \in C\left(0, T ; \mathcal{H}_{1}\right)$.

ii) Conversely, let $\sigma$ be the solution of Problem $P_{2}$. Then, there exists a unique function $u \in C^{1}(0, T ; V)$ such that (5.1) and (3.35) hold. Moreover, $u$ is the solution of Problem $P_{1}$.

Proof

i) Using (3.34) and (5.1) we obtain

$$
\langle\sigma(t), \varepsilon(w)-\varepsilon(\dot{u}(t))\rangle_{\mathcal{H}}+j(w)-j(\dot{u}(t)) \geq\langle f(t), w-\dot{u}(t)\rangle_{V} \quad \forall w \in V,
$$

for $t \in[0, T]$. Choosing $w=2 \dot{u}(t)$ and $w=0$, both in $V$, in (5.2) we find

$$
\langle\sigma(t), \varepsilon(\dot{u}(t))\rangle_{\mathcal{H}}+j(\dot{u}(t))=\langle f(t), \dot{u}(t)\rangle_{V}
$$


Using now (5.2) and (5.3) we get $\sigma(t) \in \Sigma(t)$. Moreover, from (3.19) and (5.3) we deduce

$$
\langle\varepsilon(\dot{u}(t)), \tau-\sigma(t)\rangle_{\mathcal{H}} \geq 0 \quad \forall \tau \in \Sigma(t) .
$$

Using now (3.35), (5.1) and the definition of the operator $B$, (3.31), we have

$$
\varepsilon(\dot{u})=B\left(\sigma_{1} \varepsilon\left(u_{0}\right)\right) .
$$

The inequality (3.36) is now a consequence of (5.4) and (5.5). Since $\sigma(t) \in \Sigma(t)$ it now follows from (3.11), (3.20) and (5.1) that $\sigma \in C^{1}\left(0, T ; \mathcal{H}_{1}\right)$.

ii) Conversely, let $\sigma \in C\left(0, T ; \mathcal{H}_{1}\right)$ be the solution of Problem $P_{2}$ and let $z$ be given by $z=B\left(\sigma, \varepsilon\left(u_{0}\right)\right) \in C(0, T ; \mathcal{H})$. Using (3.32) and (3.36) we find, for each $t \in[0, T]$, that

$$
\langle z(t), \tau-\sigma(t)| \geq 0 \quad \forall \tau \in \Sigma(t),
$$

where

$$
\sigma(t)=\mathcal{A}(z(t))+\mathcal{G}\left(\int_{0}^{t} z(s) d s+\varepsilon\left(u_{0}\right)\right)
$$

Let $\tilde{z} \in \mathcal{H}$ be such that $\langle\tilde{z}, \varepsilon(v))\rangle_{\mathcal{H}}=0$ for all $v \in V$. Using (3.19) we deduce that $\sigma(t) \pm \tilde{z}$ belong to $\Sigma(\mathrm{t})$ and choosing $\tau=\sigma(t) \pm \tilde{z}$ in (5.6) yields $\left\langle z(t),\left.\tilde{z}\right|_{\mathcal{H}}=0\right.$, for all $t \in[0, T]$. Using Korn's inequality (3.7) implies that the set $\varepsilon(V)=\{\varepsilon(v) \mid v \in V\}$ is a closed subspace of $\mathcal{H}$. Therefore, $\varepsilon\left(V^{\perp}\right)^{\perp}=\varepsilon(V)$, where $\perp$ represents the orthogonal complement in $\mathcal{H}$. Then, $z(t) \in \varepsilon\left(V^{\perp}\right)^{\perp}$ and also $z(t) \in \varepsilon(V)$ for each $t \in[0, T]$. Thus there exists $v(t) \in V$ such that

$$
z(t)=\varepsilon(v(t)) \quad \forall t \in[0, T] .
$$

We define now $v:[0, T] \rightarrow V$ by (5.8). Using Korn's inequality again, since $z \in C(0, T ; \mathcal{H})$, we obtain that $v \in C(0, T ; V)$.

Let $u:[0, T] \rightarrow V$ be given by $u(t)=\int_{0}^{t} v(s) d s+u_{0}$. It follows from the above that $u \in C^{1}(0, T ; V)$ and also (3.35) and (5.1) hold true. The uniqueness of $u$ follows from (5.7), Lemma 3.2, (5.8) and Korn's inequality (3.7). 
We now prove that $u$ is a solution of Problem $P_{1}$. We note that, by the subdifferentiability of $j$ on $V$ and (3.8), there exists $\bar{\tau}:[0, T] \rightarrow \mathcal{H}$ such that

$$
\left\langle\bar{\tau}, \varepsilon(w)-\left.\varepsilon(\dot{u})\right|_{\mathcal{H}}+j(w)-j(\dot{u}) \geq\langle f, w-\dot{u}\rangle_{V} \quad \forall v \in V,\right.
$$

for each $t \in[0, T]$. We choose $w=2 \dot{u}$ and then $w=0$ in (5.9), since both are in $V$, and find

$$
\langle\bar{\tau}, \varepsilon(\dot{u})\rangle_{\mathcal{H}}+j(\dot{u})=\langle f, \dot{u}\rangle_{V} .
$$

Using (5.9) and (5.10) again, we obtain $\bar{\tau} \in \Sigma(t)$. Therefore, by (3.36), (5.7), (5.8) and the above arguments we get

$$
\langle\bar{\tau}, \varepsilon(\dot{u})\rangle_{\mathcal{H}} \geq\langle\sigma, \varepsilon(\dot{u})\rangle_{\mathcal{H}}
$$

for each $t \in[0, T]$. Using now (5) and (5.11) it follows that

$$
\langle f, \dot{u}\rangle_{V} \geq\langle\sigma, \varepsilon(\dot{u})\rangle_{\mathcal{H}}+j(\dot{u}),
$$

and since the converse inequality follows from the fact that $\sigma(t) \in \Sigma(t)$, for each $t \in[0, T]$, we obtain (5.3) which, in turn, implies (5.2). The inequality (3.34) follows now from (5.1) and (5.2). This concludes the proof of Theorem 4.4 .

\section{CONTINUOUS DEPENDENCE ON THE FRICTION YIELD LIMIT}

We show that the solution of the variational problems $P_{1}$ and $P_{2}$ depend continuously on the friction yield limit $g$. The main results in this section is the following.

THEOREM 6.1 Assume that (3.9)-(3.12) and (3.14) hold. Let $u_{i}, \sigma_{i}$ be the solutions of the variational problems $P_{1}$ and $P_{2}$, respectively, with $g_{i}$ which satisfy (3.13), $i=1,2$. Then, there exists $C>0$, which depends on $\Omega, \Gamma, \mathcal{A}, \mathcal{G}$ and $T$, such that

$$
\begin{aligned}
& \left|u_{1}-u_{2}\right|_{C^{1}(0, T ; V)} \leq C\left|g_{1}-g_{2}\right|_{L^{\infty}\left(\Gamma_{C}\right)}, \\
& \left|\sigma_{1}-\sigma_{2}\right|_{C\left(0, T ; \mathcal{H}_{1}\right)} \leq C\left|g_{1}-g_{2}\right|_{L^{\infty}\left(\Gamma_{C}\right)} .
\end{aligned}
$$

In addition to the mathematical interest in this result, Theorem 6.1 is important in mechanical applications since it shows that small 
inaccuracies or variations in the friction yield limit lead to small changes in the solutions for both problems $P_{1}$ and $P_{2}$.

As an application to Theorem 6.1 we consider a physical setting where a viscoelastic part or component of a system is being acted upon by tractions on a part of its surface and is in bilateral frictional contact with a moving harder element. If we assume that the friction yield limit $g$ is a Lipschitz continuous function with respect to $v^{*}$, the velocity of the element, then Theorem 6.1 guarantees that the solution of the problem depends on $v^{*}$ Lipschitz continuously.

Proof We use the notation $j_{i}$, where here and below $i=1,2$, for the functional defined by (3.17) with $g=g_{i}$. Therefore,

$$
j_{i}(v)=\int_{\Gamma_{C}} g_{i}\left|v_{\tau}\right| d a \quad \forall v \in V .
$$

Using (3.34), for $i=1,2$, we deduce

$$
\begin{aligned}
\left\langle A\left(\varepsilon\left(\dot{u}_{i}\right)\right), \varepsilon(w)-\varepsilon\left(\dot{u}_{i}\right)\right\rangle_{\mathcal{H}} & +\left\langle\mathcal{G}\left(\varepsilon\left(u_{i}\right)\right), \varepsilon(w)-\varepsilon\left(\dot{u}_{i}\right)\right\rangle_{\mathcal{H}} \\
& +j_{i}(w)-j_{i}\left(\dot{u}_{i}\right) \geq\left\langle f, w-\dot{u}_{i}\right\rangle_{V} \quad \forall w \in V,
\end{aligned}
$$

for each $t \in[0, T]$. Then, we choose $w=\dot{u}_{2}$ in the expression for $i=1$, and $w=\dot{u}_{1}$ in the expression for $i=2$, we add the expressions that were thus obtained and we obtain

$$
\begin{aligned}
\left\langle A\left(\varepsilon\left(\dot{u}_{1}\right)\right)-\mathcal{A}\left(\varepsilon\left(\dot{u}_{2}\right)\right), \varepsilon\left(\dot{u}_{1}\right)-\left.\varepsilon\left(\dot{u}_{2}\right)\right|_{\mathcal{H}} \leq\right. & \left\langle\mathcal{G}\left(\varepsilon\left(u_{1}\right)\right)-\mathcal{G}\left(\varepsilon\left(u_{2}\right)\right), \varepsilon\left(\dot{u}_{1}\right)-\varepsilon\left(\dot{u}_{2}\right)\right\rangle_{\mathcal{H}} \\
& +j_{1}\left(\dot{u}_{2}\right)-j_{1}\left(\dot{u}_{1}\right)+j_{2}\left(\dot{u}_{1}\right)-j_{2}\left(\dot{u}_{2}\right) .
\end{aligned}
$$

Using now (3.9) and (3.7) we deduce

$$
C\left|\dot{u}_{1}-\dot{u}_{2}\right|_{V}^{2} \leq\left\langle\mathcal{A}\left(\varepsilon\left(\dot{u}_{1}\right)\right)-\mathcal{A}\left(\varepsilon\left(\dot{u}_{2}\right)\right), \varepsilon\left(\dot{u}_{1}\right)-\left.\varepsilon\left(\dot{u}_{2}\right)\right|_{\mathcal{H}},\right.
$$

and, by (3.10) we obtain

$$
\left\langle\mathcal{G}\left(\varepsilon\left(u_{1}\right)\right)-\mathcal{G}\left(\varepsilon\left(u_{2}\right)\right), \varepsilon\left(\dot{u}_{1}\right)-\left.\varepsilon\left(\dot{u}_{2}\right)\right|_{\mathcal{H}} \leq C\left|u_{1}-u_{2}\right|_{V}\left|\dot{u}_{1}-\dot{u}_{2}\right|_{V} .\right.
$$

Finally, using (6.3) it follows that

$$
j_{1}\left(\dot{u}_{2}\right)-j_{1}\left(\dot{u}_{1}\right)+j_{2}\left(\dot{u}_{1}\right)-j_{2}\left(\dot{u}_{2}\right) \leq C\left|g_{1}-g_{2}\right|_{L^{\infty}\left(\Gamma_{C}\right)}\left|\dot{u}_{1}-\dot{u}_{2}\right|_{V} .
$$


Therefore, by (6.4)-(6.7) we get

$$
\left|\dot{u}_{1}-\dot{u}_{2}\right|_{V} \leq C\left(\left|u_{1}-u_{2}\right|_{V}+\left|g_{1}-g_{2}\right|_{L^{\infty}\left(\Gamma_{C}\right)}\right)
$$

for each $t \in[0, T]$. Using now (3.35) we have

$$
u_{i}(t)=\int_{0}^{t} \dot{u}_{i}(s) d s+u_{0}
$$

and by $(6.8)$

$$
\left|\dot{u}_{1}(t)-\dot{u}_{2}(t)\right|_{V} \leq C\left(\int_{0}^{t}\left|\dot{u}_{1}(s)-\dot{u}_{2}(s)\right|_{V}+\left|g_{1}-g_{2}\right|_{L^{\infty}\left(\Gamma_{C}\right)}\right) .
$$

By applying Gronwall's inequality to (6.10) we conclude

$$
\left|\dot{u}_{1}(t)-\dot{u}_{2}(t)\right|_{V} \leq C\left|g_{1}-g_{2}\right|_{L^{\infty}\left(\Gamma_{C}\right)},
$$

for each $t \in[0, T]$. The inequality (6.1) is now a consequence of (6.9) and (6.11).

In order to prove (6.2) we note that Theorem 5.1 implies

$$
\sigma_{i}=\mathcal{A}\left(\varepsilon\left(\dot{u}_{i}\right)\right)+\mathcal{G}\left(\varepsilon\left(u_{i}\right)\right),
$$

and by (3.20) we deduce

$$
\operatorname{div} \sigma_{i}+\mathrm{f}_{0}(t)=0 \quad \text { in } \Omega, \quad t \in[0, T] .
$$

The inequality (6.2) is now a consequence of (3.9), (3.10), (6.1), (6.12) and (6.13).

\section{References}

[1] A. Amassad and M. Sofonea (1998). Analysis of a quasistatic viscoplastic problem involving Tresca friction law. Discrete and Continuous Dynamical Systems, 4(1), 55-72.

[2] A. Amassad and M. Sofonea (1998). Analysis of some evolution systems arising in rate-type viscoplasticity. In: W. Chen and S. Hu (Eds.), Dynamical Systems and Differential Equations, Discrete and Continuous Dynamical Systems, pp. 58-71.

[3] A. Amassad, M. Shillor and M. Sofonea (1999). A quasistatic contact problem for an elastic perfectly plastic body with Tresca's friction. Nonlin. Anal., 35, 95-109.

[4] L.E. Andersson (1991). A quasistatic fricional problem with normal compliance. Nonlin. Anal., 16(4), 347-369.

[5] M. Cocu (1984). Existence of solution of Signorini problems with friction. Int. J. Engng. Sci., 22, 567-581.

[6] M. Cocu, E. Pratt and M. Raous (1996). Formulation and approximation of quasistatic frictional contact. Int. J. Engng. Sci., 34(7), 783-798. 
[7] G. Duvant (1982). Loi de frottement non locale. J. Méc. Thé. Appl. Special issue, 73-78.

[8] G. Duvant and J.L. Lions (1972). Les Inéquations en. Mécanique et en Physique. Dunod, Paris.

[9] I. Hlaváček and J. Nečas (1981). Mathematical Theory of Elastic and Elastoplastic Bodies: An introduction. Elsevier, Amsterdam.

[10] I.R. Ionescu and M. Sofonea (1993). Functional and Numerical Methods in Viscoplasticity. Oxford University Press, Oxford.

[11] N. Kikuchi and T.J. Oden (1988). Contact Problems in Elasticity: A Study of Variational Inequalities and Finite Element Methods. SIAM, Philadelphia.

[12] A. Klarbring, A. Mikelic and M. Shillor (1991). A global existence result for the quasistatic frictional contact problem with normal compliance. In: G. Del Piero and F. Maceri (Eds.), Unilateral Problems in Structural Mechanics IV. pp. 85-111. Birkhaüser, Boston.

[13] P.D. Panagiotopoulos (1985). Inequality Problem in Mechanics and Applications. Birkhaüser, Basel.

[14] M. Rochdi and M. Shillor. Existence and uniqueness for a quasistatic frictional bilateral contact problem in thermoviscoelasticity. Quart. Appl. Math., to appear.

[15] M. Rochdi M. Shillor and M. Sofonea (1998). Quasistatic viscoelastic contact with normal compliance and friction. J. Elasticity, 51, 105-126.

[16] M. Shillor and M. Sofonea (2000). A quasistatic viscoelastic contact problem with friction. Int. J. Engng. Sci., 38, 1517-1533. 